\title{
Dose-Dense Chemotherapy in Metastatic Breast Cancer: Shortening the Time Interval for a Better Therapeutic Index
}

\author{
Marcus Schmidt \\ Department of Obstetrics and Gynecology, Johannes Gutenberg-University, Mainz, Germany
}

\author{
Keywords \\ Dose-dense - Metastatic · Breast cancer - Weekly . \\ Metronomic
}

\section{Summary}

Despite the advancement of targeted therapies in metastatic breast cancer, chemotherapy is still of pivotal importance. The concept of dose density is known to increase the efficacy of chemotherapy. In metastatic disease, preservation of the quality of life is equally important. Because of this, weekly regimens are a cornerstone in metastatic disease. Taxanes like paclitaxel or nab-paclitaxel as well as antracyclines are often used in palliative treatment. Further advances to increase dose density have led to the concept of daily metronomic schedules with oral chemotherapeutic drugs like cyclophosphamide, capecitabine, or vinorelbine. Metronomic chemotherapy affects tumor angiogenesis and also weakens immunosuppressive regulatory $\mathrm{T}$ cells, promoting better control of tumor progression. Weekly or daily dose-dense regimens are a reasonable compromise between efficacy and toxicity to improve the therapeutic index. This is most important for the treatment of chronic disease where palliation and preservation of quality of life are vital.

(C) 2015 S. Karger GmbH, Freiburg

\section{Introduction}

Metastatic breast cancer is a chronic disease which is treatable but still generally not curable [1]. In the last decade, there have been substantial advancements due to the increasing use of targeted therapies in estrogen receptor- or human epidermal growth factor receptor 2 (HER2)-positive advanced breast cancer. Still, despite these advances in targeted therapies, cytotoxic chemotherapy remains a cornerstone in the treatment of advanced disease.

Clearly, chemotherapy has an essential role in rapidly progressive life-threatening disease. In these situations, it is sometimes prudent to sacrifice toxicity for improved efficacy leading to rapid responses which in turn should improve the quality of life of the patient.

This goal can be achieved by combining chemotherapy with targeted agents like bevacicumab [2] and trastuzumab [3]. Another possibility to achieve a fast response is to combine several chemotherapeutic agents. Even though this approach does not lead to improved survival compared to sequential single-agent chemotherapy, it is sometimes necessary for prompt symptom control. In a meta-analysis of 12 trials with 2,317 patients randomized to combination chemotherapy compared to the same drugs used sequentially, the authors showed that combination chemotherapy led to a higher risk ratio (RR) of 1.13 (95\% confidence interval (CI) 1.03-1.24; $\mathrm{p}=0.008$ ) at the cost of a higher rate of febrile neutropenia (RR 1.32, 95\% CI 1.06-1.65; $\mathrm{p}=0.01$ ). Despite the higher response rate, there was no difference in overall survival (OS) between these treatment strategies (hazard ratio (HR) 1.04, 95\% CI 0.93-1.16; $\mathrm{p}=0.45$ ). Furthermore, combination chemotherapy led to a higher risk of progression (HR 1.16, 95\% CI 1.03-1.31; $\mathrm{p}=0.01$ ). This high-level evidence further supports the recommendations to use sequential monotherapy unless there is rapid disease progression [4].

However, it is in this very situation that the concept of dose density can come into play, including in advanced and metastatic breast cancer.

\section{Dose-Dense Chemotherapy}

In an attempt to increase the efficacy of anthracycline- and taxane-containing regimens originally used in the adjuvant treatment

\section{KARGER

\section{() 2016 S. Karger GmbH, Freiburg}

$1661-3791 / 16 / 0111-0022 \$ 39.50 / 0$ 
of breast cancer, the concept of dose-dense chemotherapy received increasing attention. Dose-dense chemotherapy increases the dose intensity of the regimen by delivering standard-dose chemotherapy at shorter intervals between cycles.

The theoretical basis of dose-dense chemotherapy is the Gompertzian model of breast cancer growth [5]. It postulates that the doubling time of breast cancer is not constant but rather increases with increasing tumor size, up to a certain volume. Thus, the Norton-Simon model predicts that the best way to destroy a heterogeneous mix of cancer cells is to eradicate the numerically dominant, faster growing cells first, followed by eradication of the more slow-growing resistant cells, leading to the concept of sequential therapy [6]. Additionally, reducing the time available for tumor regrowth (increasing dose density) may have a greater impact on clinical outcome than dose escalation. This finally led to the concept of dose-dense chemotherapy which is now commonly used in early breast cancer. In comparison to the abundant evidence on dose-dense chemotherapy in early breast cancer [7], trials using dose-dense chemotherapy requiring granulocyte-colonystimulating factors (G-CSF) to hasten hematopoietic recovery are scarce in advanced breast cancer. In a randomized phase II study, Fountzilas et al. [8] failed to demonstrate a significant difference in overall response rate ( 55 vs. $42 \%$; $p=0.10$ ) between dose-dense sequential administration of epirubicin and paclitaxel compared with combination chemotherapy every 3 weeks, even though the sequential treatment resulted in a significantly higher complete response rate ( 21.5 vs. $9 \% ; \mathrm{p}=0.02)$. Time to progression (TTP) and OS were similar. In an attempt to compare both possibilities of increasing dose intensity, namely dose escalation or dose density, another randomized phase II study assessed the efficacy and safety of the 2 different approaches. The authors compared epirubicin 110 $\mathrm{mg} / \mathrm{m}^{2}$ combined with paclitaxel $200 \mathrm{mg} / \mathrm{m}^{2}$ every 21 days and epirubicin $75 \mathrm{mg} / \mathrm{m}^{2}$ combined with paclitaxel $175 \mathrm{mg} / \mathrm{m}^{2}$ every 10 days, both supported with G-CSF in patients with advanced breast cancer [9]. Response rates (75 vs. 70\%), progression-free survival (PFS) (6 vs. 7 months), and OS (16 vs. 14 months) were comparable between the 2 treatment arms. Grade 3/4 toxicities were higher in the dose-escalated compared to the dose-dense arm (39 vs. 29\%). The authors concluded that the dose-dense regimen seemed to be the preferred schedule due to fewer side effects. However, phase III trials of dose-dense chemotherapy with G-CSF support in the advanced breast cancer setting are missing. Due to this important limitation, dose-dense regimens given every 2 weeks and requiring G-CSF support do not play a relevant role in the treatment of metastatic breast cancer.

However, dose density can be increased by further shortening the time intervals between cycles. If the time interval is reduced to 1 week, a disproportionate reduction in chemotherapy dosage increases drug intensity. This concept allows for abandonment of G-CSF with at least sustained efficacy. What makes this idea particularly promising in metastatic breast cancer is the fact that less toxicity should lead to improved quality of life which is a central goal in the treatment of advanced disease.

Dose-Dense Chemotherapy in Metastatic Breast Cancer

\section{Weekly Schedules}

Seidmann et al. [10] evaluated weekly 1-h infusions of paclitaxel in 16 pretreated patients with metastatic breast cancer. Paclitaxel was administered weekly at a starting dose of $100 \mathrm{mg} / \mathrm{m}^{2}$ continuing until disease progression or onset of intolerable toxicity. No episodes of febrile neutropenia or acute hypersensitivity reactions were noted. However, some degree of cumulative peripheral neuropathy was observed in 3 patients. 6 patients obtained either a partial or a complete response, 4 had a minor response, and 5 patients showed stable disease. The authors concluded that despite the potential neurotoxicity, the high therapeutic index, manageable toxicity, and convenient administration schedule made this weekly dose-dense regimen an attractive treatment alternative for patients with metastatic breast cancer. Building on these promising early results, this group of researchers reported a phase II study with 30 patients with metastatic breast cancer receiving sustained weekly paclitaxel therapy at an initial dose of $100 \mathrm{mg} / \mathrm{m}^{2}$ until disease progression [11]. The overall response rate was $53 \%$ with median response duration of 7.5 months. Again, febrile neutropenia was not observed, and only 2 cases of grade 3 neutropenia were reported. These promising results led the authors to conclude that weekly therapy should be considered as a current clinical option for patients with metastatic breast cancer and should be incorporated into future comparative clinical trials. Indeed, numerous largely small trials were undertaken to examine the efficacy and side effects of weekly paclitaxel. Lück and Roché [12] reviewed those early trials showing response rates of up to $86 \%$ with single-agent therapy and up to $87 \%$ with combination therapy. In a multicenter phase II trial, Perez et al. [13] treated 212 metastatic breast cancer patients with weekly paclitaxel. Up to 2 prior chemotherapy regimens for metastatic disease were allowed. Paclitaxel $80 \mathrm{mg} / \mathrm{m}^{2}$ was administered weekly for 4 weeks per 4 -week cycle. The overall response rate was $21.5 \%$, with $41.8 \%$ of patients having disease stabilization. Median TTP was 4.7 months, and OS was 12.8 months. $9 \%$ of patients developed grade 3 neurotoxicity. Patients $\geq 65$ years of age had an equivalent overall response rate and no greater incidence of toxicity compared with younger patients. Quality of life was maintained relatively well in patients treated with weekly paclitaxel. All in all, therapy was well tolerated and demonstrated reasonable activity in pretreated patients with advanced disease. Finally, a phase III study of weekly paclitaxel was published [14]. Patients with metastatic breast cancer were randomly assigned to paclitaxel $175 \mathrm{mg} / \mathrm{m}^{2}$ every 3 weeks or paclitaxel $80 \mathrm{mg} / \mathrm{m}^{2}$ weekly. HER2-positive patients received additional trastuzumab; HER2negative patients were randomly assigned for trastuzumab in addition to paclitaxel. Therapy was continued until disease progression or limiting toxicity. 735 patients were finally analyzed. The response rate was 42 vs. $29 \%$ (odds ratio (OR) 1.75; p = 0.0004), median TTP was 9 vs. 5 months (HR 1.43; p < 0.0001), and median survival was 24 vs. 12 months (HR 1.28; $\mathrm{p}=0.0092$ ). For HER2negative patients, trastuzumab did not improve efficacy. Grade 3 neuropathy was more common with weekly dosing (24 vs. $12 \%$; $\mathrm{p}=0.0003$ ). This large phase III trial confirmed the superior effi- 
cacy of dose-dense weekly paclitaxel with neurotoxicity as treatment-limiting toxicity.

Surprisingly, this superiority of weekly administration was not seen for docetaxel [15]. In their phase III trial, metastatic breast cancer patients treated with docetaxel every 3 weeks had a higher response rate but experienced similar PFS and OS and more pronounced toxicity as compared to patients who received weekly docetaxel.

A potential advancement of taxane-based therapy comes in the form of nanoparticle albumin-bound (nab)-paclitaxel. In a phase III trial, nab-paclitaxel demonstrated a significantly higher overall response rate, significantly longer TTP, and significantly greater OS in metastatic breast cancer patients treated with second-line or greater therapy compared with patients who received conventional paclitaxel every 3 weeks (q3w) [16]. Building on these encouraging results, Gradishar et al. [16] compared in a phase II trial nab-paclitaxel $300 \mathrm{mg} / \mathrm{m}^{2} \mathrm{q} 3 \mathrm{w}, 100 \mathrm{mg} / \mathrm{m}^{2}$ weekly, or $150 \mathrm{mg} / \mathrm{m}^{2}$ weekly, or docetaxel $100 \mathrm{mg} / \mathrm{m}^{2} \mathrm{q} 3 \mathrm{w}$. This trial demonstrated superior efficacy and safety of weekly nab-paclitaxel compared with docetaxel in 302 patients with metastatic breast cancer in the first-line setting. The disease control rate was significantly higher for patients receiving weekly nab-paclitaxel compared with docetaxel. Grade 3/4 fatigue, neutropenia, and febrile neutropenia were less frequent in all nab-paclitaxel arms. Frequency and grade of peripheral neuropathy were comparable. Again, these findings point to an increased efficacy for dose-dense weekly application of taxanes.

Clearly, the concept of dose density in metastatic disease applies to anthracyclines also. Epirubicin as well as doxorubicin on a weekly basis achieved responses with a good safety profile [17]. However, one has to keep in mind that a substantial proportion of breast cancer patients will already have received anthracyclines in the adjuvant setting, and the well-documented cumulative cardiotoxicity of anthracyclines is an issue in the advanced setting when thinking about conventional dose-dense anthracyclines. A possible approach to circumvent this problem of cumulative cardiotoxicity is the use of pegylated liposomal doxorubicin (PLD) which was effective and well tolerated without cardiac events in patients with metastatic breast cancer who had received prior adjuvant anthracycline-containing chemotherapy [18]. Considering dose density, PLD was shown to be effective with little side effects when used dose-dense and bi-weekly $\left(20 \mathrm{mg} / \mathrm{m}^{2}\right)$ in elderly patients with advanced breast cancer [19]. Most importantly, no cardiac events were registered in this study. Using a similar dose-dense schedule, Munzone et al. [20] reported a clinical benefit rate of $45 \%$ without any cardiac events in 52 patients with advanced breast cancer.

\section{Metronomic Schedules}

For practical reasons, it is not feasible to shorten the time interval between chemotherapy cycles to below 1 week in infusion regimens. However, if using oral chemotherapy, daily administration is feasible and convenient for the patient. In recent years, this concept of so-called low-dose metronomic chemotherapy received in- creasing attention in advanced breast cancer [21]. Metronomic chemotherapy was shown to induce disease control in patients with advanced-stage breast cancer with a low incidence of adverse events and a good safety profile. In a recent systematic review, Montagna et al. [22] underlined that metronomic chemotherapy compromises the repair process of endothelial cells, leading to an anti-angiogenic effect. In addition to this anti-angiogenic action, metronomic chemotherapy could exert an immunological effect through restoration of the anticancer properties of the immune system and induction of tumor dormancy. The authors concluded that metronomic chemotherapy in metastatic breast cancer has a low toxicity profile with good efficacy, and also has potentially significant advantages with regard to healthcare costs.

The backbone of metronomic regimens in advanced breast cancer is cyclophosphamide, given mostly at a fixed dose of $50 \mathrm{mg}$ daily. A comparative analysis showed absent or moderate hematologic and intestinal toxicity of metronomic as opposed to maximum-tolerated-dose chemotherapy [23]. Low-dose metronomic chemotherapy is usually given over a prolonged period of time. Because of this, it could be suspected that altered pharmacokinetic characteristics due to long-term drug exposure might potentially result in acquired resistance and increased risk of unfavorable drug interactions. However, it was shown in a comprehensive preclinical pharmacokinetic and pharmacodynamic study that exposure of mice for $\geq 8$ weeks did not compromise subsequent activity of metronomic chemotherapy [24].

Numerous combination partners of metronomic cyclophosphamide have since been investigated in breast cancer, mostly in phase II trials. A combination of metronomic cyclophosphamide with oral methotrexate showed promising results in heavily pretreated advanced breast cancer patients with a clinical benefit rate of $31.7 \%$ and only scarce toxicity [25]. In further studies of this group, metronomic cyclophosphamide and methotrexate were combined with thalidomide. This combination failed to improve clinical efficacy. However, metronomic cyclophosphamide and methotrexate achieved in this study an impressive clinical benefit rate of $41.5 \%$ with a median TTP of 3.8 months [26]. A combined analysis of these 2 trials showed that metronomic chemotherapy administered for a prolonged time was feasible and provided long-term clinical benefit in a clinically significant proportion of patients, without significant toxicity despite prolonged use [27]. Furthermore, a comparative pharmacoeconomic evaluation showed that low-dose metronomic chemotherapy was a cost-effective/cost-saving therapy for palliative treatment of metastatic breast cancer patients [28].

The main targets of low-dose metronomic chemotherapy are the endothelial cells of the growing vasculature of a tumor, making it an anti-angiogenic therapy [29]. In vitro results were consistent with the notion that continuous low-dose therapy with various chemotherapeutic drugs might have a highly selective effect against cycling vascular endothelial cells. This in turn might be relevant for the use of continuous or frequent administration of low doses of certain chemotherapeutic drugs as an optimal way of delivering anti-angiogenic therapy [30]. Taken together, the anti-angiogenic 
basis of metronomic chemotherapy involves the growth arrest or apoptosis of activated endothelial cells in the tumor vasculature, sustained suppression of mobilization of endothelial progenitor cells, and the induction of endogenous inhibitors of angiogenesis such as thrombospondin-1 [31].

Because of these clearly demonstrated anti-angiogenic properties of low-dose metronomic chemotherapy, combination with other agents targeting angiogenesis seems a promising concept. Indeed, the combination of metronomic chemotherapy (daily lowdose cyclophosphamide + capecitabine) with bevacizumab proved highly efficacious in heavily pretreated advanced breast cancer patients. A phase II trial showed in 46 patients a clinical benefit rate of $68 \%$ and a median TTP of 42 weeks with a mild toxicity profile [32]. Higher baseline levels of circulating endothelial cells were correlated with improved outcome.

In addition to the well-documented anti-angiogenic properties of metronomic chemotherapy, reduction of regulatory $\mathrm{T}$ cells (Tregs) might contribute to the beneficial effects of metronomic chemotherapy containing cyclophosphamide [33]. Therefore, metronomic cyclophosphamide does not only affect tumor angiogenesis but also strongly weakens immunosuppressive Tregs, promoting better control of tumor progression. Because of the unfavorable influence of Tregs on breast cancer, strategies to reduce this $\mathrm{T}$ cell subset in breast cancer patients are important. Indeed, metronomic cyclophosphamide in advanced cancer patients induced a profound and selective reduction of circulating Tregs, associated with suppression of their inhibitory functions on conventional $\mathrm{T}$ cells and natural killer cells leading to a restoration of peripheral $\mathrm{T}$ cell proliferation and innate killing activities [33]. Importantly, only low doses of cyclophosphamide could achieve a selective Treg depletion, while higher doses induced a profound decrease in the number of circulating lymphocytes, an effect observed on all lymphocyte subpopulations without any selective activity on Tregs [33]. Interestingly, in a small exploratory study investigating $12 \mathrm{pa}-$ tients with metastatic breast cancer treated with metronomic cyclophosphamide $50 \mathrm{mg}$ daily, Treg depletion coincided with a strong increase in breast tumor-reactive $\mathrm{T}$ cells $(\mathrm{p}=0.03)$ [34]. Tumor-reactive $\mathrm{T}$ cells but not Tregs correlated with disease stabilization $(\mathrm{p}=0.03)$ and $\mathrm{OS}(\mathrm{p}=0.027)$.

Taken together, these data suggest that metronomic chemotherapy is an effective and feasible therapeutic option for advanced breast cancer. Clearly, other oral drugs with proven efficacy in advanced breast cancer like capecitabine or oral vinorelbine are also promising candidates for metronomic therapy.

In a phase II trial, 60 patients with metastatic breast cancer received continuous metronomic capecitabine monotherapy $(1,500$ mg once daily) [35]. The clinical benefit rate was $62 \%$. Median TTP and OS were 7 and 17 months, respectively. Tolerability was excellent with little side effects. Oral vinorelbine was also tested in a metronomic schedule for the first-line treatment of elderly patients with metastatic breast cancer [36]. In this study, 34 patients (median age 74 years) were treated with oral vinorelbine at $70 \mathrm{mg} / \mathrm{m}^{2}$, fractionated on days 1,3 , and 5, 3 weeks on/1 week off, every 4 weeks. 2 complete and 11 partial responses were noted. PFS was 7.7 months and OS 15.9 months. In their conclusion, the authors underlined that this well-tolerated regimen merits further investigation in combination with other chemotherapy agents. Thus, a reasonable next step in the development of metronomic regimens in metastatic breast cancer could be the combination of oral vinorelbine and capecitabine.

Indeed, in a phase I trial enrolling 36 patients with advanced breast cancer, the combination of oral metronomic vinorelbine with capecitabine was examined. The recommended maximum tolerated doses were vinorelbine $60 \mathrm{mg}$ every other day and capecitabine $1,250 \mathrm{mg} / \mathrm{m}^{2}$ days $1-14$ every 21 days. This trial showed that this metronomic combination was a well-tolerated and feasible regimen with 2 complete and 10 partial responses documented [37]. In the phase I/II VICTOR-1 study, the combination of metronomic vinorelbine thrice weekly and capecitabine $500 \mathrm{mg}$ thrice daily was investigated [38]. The dosage of vinorelbine could be increased up to $40 \mathrm{mg}$ thrice weekly. 31 patients evaluable for efficacy had a clinical benefit rate of $58.1 \%$. This combination comes close to the original concept of metronomic and dose-dense chemotherapy with capecitabine administered daily. However, oral vinorelbine is given only thrice per week. Bearing this in mind, this schedule is not truly metronomic in the narrower sense.

In an effort to further develop the dose-dense potential of truly metronomic oral vinorelbine, the breast cancer study group of the Arbeitsgemeinschaft Gynäkologische Onkologie (AGO-B) is planning the VinoMetro trial in metastatic breast cancer. In this multicenter phase II trial, patients with metastatic hormone receptorpositive and HER2-negative breast cancer resistant to endocrine therapy will be enrolled and treated with oral vinorelbine administered at a daily dose of $30 \mathrm{mg}$ without breaks. The primary objective is the clinical benefit rate at 24 weeks after start of treatment. A comprehensive translational research program investigating antiangiogenic and immunomodulatory effects of this metronomic schedule is accompanying this trial.

In conclusion, the concept of dose density has an important role in the treatment of metastatic breast cancer. Since the therapeutic goals in metastatic breast cancer inevitably differ from those in early breast cancer, increasing attention is directed towards less toxic, albeit effective, weekly or even metronomic regimens. This in turn should lead to an improved therapeutic index in this chronic disease.

\section{Disclosure Statement}

Prof. Dr. Marcus Schmidt is a named inventor on patent applications regarding prediction of chemotherapeutic response in breast cancer and molecular markers for breast cancer prognosis. He is a consultant for Pfizer, Roche, Eisai, Celgene, Novartis, Pierre-Fabre, TEVA, and Sividon, and has received honoraria from AstraZeneca, Novartis, Pfizer, Celgene, Pierre-Fabre, GlaxoSmithKline, Eisai, Roche, Sividon, TEVA, and Amgen. He has previously received funding from Bayer Healthcare AG, Sanofi-Aventis, and the Bundesministerium für Bildung und Forschung (BMBF). 


\section{References}

1 Cardoso F, Costa A, Norton L, et al.: ESO-ESMO 2nd international consensus guidelines for advanced breas cancer (ABC2)dagger. Ann Oncol 2014;25:1871-1888.

2 Miller K, Wang M, Gralow J, et al.: Paclitaxel plus bevacizumab versus paclitaxel alone for metastatic breast cancer. N Engl J Med 2007;357:2666-2676.

3 Slamon DJ, Leyland-Jones B, Shak S, et al.: Use of chemotherapy plus a monoclonal antibody against HER2 for metastatic breast cancer that overexpresses HER2. N Engl J Med 2001;344:783-792.

4 Dear RF, McGeechan K, Jenkins MC, et al.: Combination versus sequential single agent chemotherapy for metastatic breast cancer. Cochrane Database Syst Rev 2013; 12:CD008792.

5 Norton L: A Gompertzian model of human breast cancer growth. Cancer Res 1988;48:7067-7071.

6 Norton L: Evolving concepts in the systemic drug therapy of breast cancer. Semin Oncol 1997;24:S103-S10-10

7 Petrelli F, Cabiddu M, Coinu A, et al.: Adjuvant dosedense chemotherapy in breast cancer: a systematic review and meta-analysis of randomized trials. Breast Cancer Res Treat 2015;151:251-259.

8 Fountzilas G, Papadimitriou C, Dafni U, et al.: Dosedense sequential chemotherapy with epirubicin and paclitaxel versus the combination, as first-line chemotherapy, in advanced breast cancer: a randomized study conducted by the Hellenic Cooperative Oncology Group. J Clin Oncol 2001;19:2232-2239.

9 Lalisang RI, Erdkamp FLG, Rodenburg CJ, et al.: Epirubicin and paclitaxel with G-CSF support in first line metastatic breast cancer: a randomized phase II study of dose-dense and dose-escalated chemotherapy. Breast Cancer Res Treat 2011;128:437-445.

10 Seidman AD, Hudis CA, McCaffrey J, et al.: Dosedense therapy with paclitaxel via weekly 1-hour infusion: preliminary experience in the treatment of metastatic breast cancer. Semin Oncol 1997;24:S1772-S17-76.

11 Seidman AD, Hudis CA, Albanell J, et al.: Dose-dense therapy with weekly 1-hour paclitaxel infusions in the treatment of metastatic breast cancer. J Clin Oncol 1998;16:3353-3361.

12 Luck H, Roche H: Weekly paclitaxel: an effective and well-tolerated treatment in patients with advanced breast cancer. Crit Rev Oncol Hematol 2002;44 (suppl):S15-30.

13 Perez EA, Vogel CL, Irwin DH, et al.: Multicenter phase II trial of weekly paclitaxel in women with metastatic breast cancer. J Clin Oncol 2001;19:4216-4223.

14 Seidman AD, Berry D, Cirrincione C, et al.: Randomized phase III trial of weekly compared with every3-weeks paclitaxel for metastatic breast cancer, with trastuzumab for all HER-2 overexpressors and random assignment to trastuzumab or not in HER-2 nonoverexpressors: final results of Cancer and Leukemia Group B protocol 9840. J Clin Oncol 2008;26:1642-1649.
15 Rivera E, Mejia JA, Arun BK, et al.: Phase 3 study comparing the use of docetaxel on an every-3-week versus weekly schedule in the treatment of metastatic breast cancer. Cancer 2008;112:1455-1461.

16 Gradishar WJ, Tjulandin S, Davidson N, et al.: Phase III trial of nanoparticle albumin-bound paclitaxel compared with polyethylated castor oil-based paclitaxel in women with breast cancer. J Clin Oncol 2005; 23:7794-7803.

17 Gasparini G, Dal Fior S, Panizzoni GA, et al.: Weekly epirubicin versus doxorubicin as second line therapy in advanced breast cancer. A randomized clinical trial. Am J Clin Oncol 1991;14:38-44.

18 Trudeau ME, Clemons MJ, Provencher L, et al.: Phase II multicenter trial of anthracycline rechallenge with pegylated liposomal doxorubicin plus cyclophosphamide for first-line therapy of metastatic breast cancer previously treated with adjuvant anthracyclines. J Clin Oncol 2009;27:5906-5910.

19 Basso U, Roma A, Brunello A, et al.: Bi-weekly liposomal doxorubicin for advanced breast cancer in elderly women ( $\geq 70$ years). J Geriatr Oncol 2013;4:340-345.

20 Munzone E, Di Pietro A, Goldhirsch A, et al.: Metronomic administration of pegylated liposomal-doxorubicin in extensively pre-treated metastatic breast cancer patients: a mono-institutional case-series report. Breast 2010;19:33-37.

21 Munzone E, Colleoni M: Clinical overview of metronomic chemotherapy in breast cancer. Nat Rev Clin Oncol 2015;12:631-644.

22 Montagna E, Cancello G, Dellapasqua S, et al.: Metronomic therapy and breast cancer: a systematic review. Cancer Treat Rev 2014;40:942-950.

23 Emmenegger U, Man S, Shaked Y, et al.: A comparative analysis of low-dose metronomic cyclophosphamide reveals absent or low-grade toxicity on tissues highly sensitive to the toxic effects of maximum tolerated dose regimens. Cancer Res 2004;64:3994-4000.

24 Emmenegger U, Shaked Y, Man S, et al.: Pharmacodynamic and pharmacokinetic study of chronic low-dose metronomic cyclophosphamide therapy in mice. Mol Cancer Ther 2007;6:2280-2289.

25 Colleoni M, Rocca A, Sandri MT, et al.: Low-dose oral methotrexate and cyclophosphamide in metastatic breast cancer: antitumor activity and correlation with vascular endothelial growth factor levels. Ann Oncol 2002;13:73-80

26 Colleoni M, Orlando L, Sanna G, et al.: Metronomic low-dose oral cyclophosphamide and methotrexate plus or minus thalidomide in metastatic breast cancer: antitumor activity and biological effects. Ann Oncol $2006 ; 17: 232-238$
27 Orlando L, Cardillo A, Rocca A, et al.: Prolonged clinical benefit with metronomic chemotherapy in patients with metastatic breast cancer. Anticancer Drugs 2006 17:961-967

28 Bocci G, Tuccori M, Emmenegger U, et al.: Cyclophosphamide-methotrexate 'metronomic' chemotherapy for the palliative treatment of metastatic breast cancer. A comparative pharmacoeconomic evaluation. Ann Oncol 2005; 16:1243-1252.

29 Browder T, Butterfield CE, Kraling BM, et al.: Antiangiogenic scheduling of chemotherapy improves efficacy against experimental drug-resistant cancer. Cancer Res 2000;60:1878-1886.

30 Bocci G, Nicolaou KC, Kerbel RS: Protracted low-dose effects on human endothelial cell proliferation and survival in vitro reveal a selective antiangiogenic window for various chemotherapeutic drugs. Cancer Res 2002;62:6938-6943.

31 Kerbel RS, Kamen BA: The anti-angiogenic basis of metronomic chemotherapy. Nat Rev Cancer 2004; 423-436.

32 Dellapasqua S, Bertolini F, Bagnardi V, et al.: Metronomic cyclophosphamide and capecitabine combined with bevacizumab in advanced breast cancer. J Clin Oncol 2008;26:4899-4905.

33 Ghiringhelli F, Menard C, Puig PE, et al.: Metronomic cyclophosphamide regimen selectively depletes $\mathrm{CD} 4+\mathrm{CD} 25+$ regulatory $\mathrm{T}$ cells and restores $\mathrm{T}$ and $\mathrm{NK}$ effector functions in end stage cancer patients. Cancer Immunol Immunother 2007;56:641-648.

34 Ge Y, Domschke C, Stoiber N, et al.: Metronomic cyclophosphamide treatment in metastasized breast cancer patients: immunological effects and clinical outcome. Cancer Immunol Immunother 2012;61:353-362.

35 Fedele P, Marino A, Orlando L, et al.: Efficacy and safety of low-dose metronomic chemotherapy with capecitabine in heavily pretreated patients with metastatic breast cancer. Eur J Cancer 2012;48:24-29.

36 Addeo R, Sgambato A, Cennamo G, et al.: Low-dose metronomic oral administration of vinorelbine in the first-line treatment of elderly patients with metastatic breast cancer. Clin Breast Cancer 2010;10:301-306.

37 Saridaki Z, Malamos N, Kourakos P, et al.: A phase I trial of oral metronomic vinorelbine plus capecitabine in patients with metastatic breast cancer. Cancer Chemother Pharmacol 2012;69:35-42.

38 Cazzaniga ME, Torri V, Villa F, et al.: Efficacy and safety of the all-oral schedule of metronomic vinorelbine and capecitabine in locally advanced or metastatic breast cancer patients: the phase I-II VICTOR-1 study. Int J Breast Cancer 2014;2014:769790. 